\title{
HYPERELLIPTIC COMPACT NON-ORIENTABLE KLEIN SURFACES WITHOUT BOUNDARY
}

\author{
By J. A. BUJALANCE*
}

By a compact non-orientable Klein surface (KS) $X$, we shall mean a compact non-orientable surface together with a dianalytic structure on $X$ [3].

A dianalytic homeomorphism of $X$ onto itself will be called an automorphism. We say that a compact non-orientable $\mathrm{KS}$ without boundary $X$ is $q$-hyperelliptic if and only is there exists an involution $\Phi$ of $X$ such that $X /\langle\Phi\rangle$ has algebraic genus $q$ (if $q=0, X$ is hyperelliptic, if $q=1$, then $X$ is elliptic-hyperelliptic).

In this paper we characterize the $q$-hyperellipticity of compact non-orientable KS without boundary by means of non-Euclidean crystallographic groups (NEC groups). Similar characterizations for compact orientable KS without boundary (i. e. Riemann surfaces) or for compact KS with boundary have been obtained in [11], [6], [7], [8]. As a consequence from these results, it is obtained: The bound $84(p-1)$ for a group of automorphisms of a non orientable KS without boundary can be reduced to $12(p-1)$ for most of $q$-hyperelliptic KS.

\section{Preliminary}

In this paper we characterize KS by means of NEC groups. Such a group is a discrete subgroup of the group $G$ of all isometries of the hyperbolic plane, including orientation-reversing ones, with compact quotient space (see [10], [14]).

Each NEC group has a signature, that is

$$
\left(g ; \pm ;\left[m_{1}, \cdots, m_{t}\right] ;\left\{\left(n_{i 1} \cdots n_{\imath s_{i}}\right) i=1 \cdots k\right\}\right) .
$$

The numbers $m_{\imath}$ are the proper periods, the brachets $\left(n_{i 1} \cdots n_{\imath s_{i}}\right)$ are the periodcycles and the $n_{\imath}$, are the periods of the period-cycles. This signature determines a presentation of the group. Generators:

i) $x_{\imath} \quad i=1, \cdots, r$

ii) $e_{i} \quad i=1, \cdots, k$

iii) $c_{\imath}, i=1, \cdots, k ; j=0, \cdots, s_{\imath}$

iv) (if sign ' + ') $a_{\imath}, b_{i} \quad i=1, \cdots, g$ (if sign '-') $d_{\imath} \quad i=1, \cdots, g$

* Partially supported by CAICYT

Received April 4, 1988 
relations :

i) $x_{\imath}^{m_{\imath}}=1 \quad i=1, \cdots, r$

ii) $e_{2}^{-1} c_{i 0} e_{2} c_{2 s_{i}}=1 \quad i=1, \cdots, k$

iii) $c_{\imath \jmath-1}^{2}=c_{\imath \jmath}^{2}=\left(c_{\imath \jmath-1} c_{\imath j}\right)^{n_{\imath \jmath}=1} \quad i=1, \cdots, k ; j=1, \cdots, s_{\imath}$

iv) (if sign ' + '): $x_{1} \cdots x_{r} e_{1} \cdots e_{k} a_{1}^{-1} b_{1}^{-1} a_{1} b_{1} \cdots a_{g}^{-1} b_{g}^{-1} a_{g} b_{g}=1$

v) (if sign '-'): $x_{1} \cdots x_{r} e_{1} \cdots e_{k} d_{1}^{2} \cdots d_{g}^{2}=1$.

An NEC group $\Gamma$ with signature (1) has an associated area

$$
|\Gamma|=2 \pi\left(\alpha g+k-2+\sum_{\imath=1}^{r}\left(1-1 / m_{\imath}\right)+\frac{1}{2} \sum_{i=1}^{k} \sum_{j=1}^{s i}\left(1-1 / n_{\imath j}\right)\right),
$$

where $\alpha=1$ if the sign is ' - ' and $\alpha=2$ if sign is ' + '.

Let $X$ be a KS of topological genus $g$ with $k$ boundary components, and algebraic genus $p=\alpha g+k-1 \quad(\alpha=2$ if $X$ is orientable and $\alpha=1$ if $X$ is nonorientable). Then $X$ may be expressed as $D / \Gamma, D$ being the hyperbolic plane and $\Gamma$ an NEC group with signature

$$
\left(g ; \pm ;[-] ;\left\{(-)^{k}\right\}\right) \text {, }
$$

where the sign is ' + ' if $X$ is orientable and ' - ' if $X$ is non-orientable.

May [12] proved that a group of automorphism of the surface $D / \Gamma$, may be expressed as $\Gamma^{\prime} / \Gamma, \Gamma^{\prime}$ being another NEC group such that $\Gamma \triangleleft \Gamma^{\prime}$. The full group of automorphism of $D / \Gamma$ is $N_{G}(\Gamma) / \Gamma$, where $N_{G}(\Gamma)$ is the normalizer of $\Gamma$ in $G$. When $\Gamma^{\prime}$ and $\Gamma$ are two NEC groups such that $\left[\Gamma^{\prime}: \Gamma\right]=N$ then $N\left|\Gamma^{\prime}\right|=|\Gamma|$.

An NEC group $\Gamma$ has the canonical Fuchsian subgroup $\Gamma^{+}$associated [13]. The Riemann surface $D / \Gamma^{+}$is canonically associated to the $\mathrm{KS} D / \Gamma$.

From now on, a KS is assumed to have algebraic genus not smaller than 2 .

\section{Non-orientable $Q$-hyperelliptic surfaces.}

In this Section we characterize by means of NEC groups the compact $q$-hyperelliptic KS.

Proposition 2.1. Let $X=D / \Gamma$ be a non-orientable $K S$ without boundary. Then $X$ is q-hyperelliptic, if and only if there exists an NEC group $\Gamma_{1}$ of algebraic genus $q$, containg $\Gamma$ as a subgroup of index 2 . (The group $\Gamma_{1}$ is said to be a q-hyperellipticity group).

Proof. Let us suppose that an NEC group $\Gamma_{1}$ with algebraic genus $q$, such that $\left[\Gamma_{1}: \Gamma\right]=2$ exists. Then $\Gamma_{1} / \Gamma$ is a group of automorphisms of $D / \Gamma$ of order two, and $D / \Gamma_{1}$ has genus $q$. Thus $D / \Gamma$ is $q$-hyperelliptic.

If $X$ is $q$-hyperelliptic then the exists an involution $\Phi$ of $D / \Gamma$ isuch that $X^{\prime}=X /\langle\Phi\rangle$ has genus $q$, moreover $\langle\Phi\rangle \cong \Gamma_{1} / \Gamma$, for a certain NEC group $\Gamma_{1}$ 
such that $\left[\Gamma_{1}: \Gamma\right]=2$. Since $X^{\prime}=X /\langle\Phi\rangle \cong(D / \Gamma) /\left(\Gamma_{1} / \Gamma\right) \cong D / \Gamma_{1}$ and the genus of $X^{\prime}$ is $q$, the algebraic genus of $\Gamma_{1}$ is $q$.

THEOREM 2.2. Let $X=D / \Gamma$ be a non-orientable $K S$ without boundary with algebraic genus $p$. Then $X$ is q-hyperelliptic if and only if there exists an NEC group $\Gamma_{1}$, containing $\Gamma$ as a subgroup of index 2 and having the signature of one of the following types.

(a) $\left(h ;+;[2 \stackrel{(p+1)-2 q}{\cdots \cdots \cdots \cdots \cdots} 2] ;\left\{(-)^{q-2 h+1}\right\}\right] ; 0 \leqq h \leqq q / 2$

(b) $\left(h ;-;[2 \stackrel{(p+1)-2 q}{\cdots \cdots \cdots \cdots} 2] ;\left\{(-)^{q-h+1}\right\}\right) ; 0 \leqq h \leqq q$ if $p$ is even, $1 \leqq h \leqq q+1$ if $p$ is odd. Moreover

1) For each $0 \leqq h \leqq q / 2$ there exists a non-orientable $q$-hyperelliptıc $K S$ without boundary $D / \Gamma$ whose $q$-hyperellipticity group has signature (a).

2) For each $1 \leqq h \leqq q$ (if $p$ is even) and for each $1 \leqq h \leqq q+1$ (if $p$ is odd) there exists a non-orientable $q$-hyperelliptic $K S$ without boundary $D / \Gamma$ whose $q$-hyperellipticity group has signature (b).

Proof. Sufficiency is a consequence of Proposition 2.1.

We are going to see neccesity. Since $X=D / \Gamma$ is a non-orientable $q$-hyperelliptic KS without boundary, there exists an involution $\Phi$ of $X$, such that the algebraic genus of $X /\langle\Phi\rangle$ is $q$. There is an NEC group $\Gamma_{1}$ such that $\langle\Phi\rangle \cong \Gamma_{1} / \Gamma$.

Having in mind that the signature of $\Gamma$ is $(p+1 ;-;[-] ;\{-\})$ we are going to determine the possible signatures of $\Gamma_{1}$. Since the algebraic genus of $\Gamma_{1}$ is $q$, thus by theorem 1 [5] the possible signatures of $\Gamma_{1}$ will have the following form,

$$
\sigma\left(\Gamma_{1}\right)=\left(h ; \pm ;[2 \cdots \cdots 2] ;\left\{(-)^{q-\alpha h+1}\right\}\right),
$$

where $\alpha=2$ if the sign is ' + ' and $\alpha=1$ if sign is ' - '.

From the relation between the areas of the fundamental regions of $\Gamma$ and $\Gamma_{1}$ we have, $|\Gamma|=2\left|\Gamma_{1}\right|$. Thus $p-1=2(q-1+t / 2)$, and hence $t=p+1-2 q$. So, the signature of $\Gamma_{1}$ has the form,

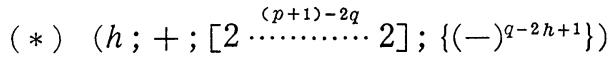

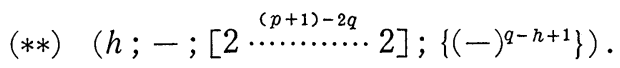

If $\Gamma_{1}$ has a signature $(*)$, then $q-2 h+1 \geqq 1$; otherwise $\Gamma_{1}$ only has orientation preserving elements. As a result $\Gamma_{1}$ could not contain the subgroup $\Gamma$, since the last one contains orientation reversing elements. So $0 \leqq h \leqq q / 2$.

If $\Gamma_{1}$ has signature $(* *)$, then $1 \leqq h \leqq q+1$. If its happen that $h=q+1$, then $\Gamma_{1}$ has a signature of the form

$$
(q+1 ;-;[2 \stackrel{(p+1)-2 h}{\cdots \cdots \cdots \cdots}])
$$


and so the $q$-hyperellipticity homeomorphism $\theta: \Gamma_{1} \rightarrow Z_{2}$ verifies $\theta\left(x_{\imath}\right)=\overline{1}$ for $i=1, \cdots,(p+1)-2 q$. Thus since $\theta$ preserves the relations of $\Gamma_{1}$ then

$$
\theta\left(d_{1}^{2} \cdots d_{q+1}^{2} x_{1} \cdots x_{(p+1)-2 q}\right)=\overline{0}
$$

consequently $\theta\left(x_{1} \cdots x_{(p+1)-2 q}\right)=\overline{0}$; and it is impossible if $p$ is even. And so if $p$ is even $h \leqq q$, and if $p$ is odd $h \leqq q+1$.

Now, we are going to see that 1 ) is satisfied. Given $h, 0 \leqq h \leqq q / 2$ and an NEC group $\Gamma_{1}$ with signature (a), we define the epimorphism $\theta: \Gamma_{1} \rightarrow Z_{2}$, by means of

$$
\begin{array}{ll}
\theta\left(a_{\imath}\right)=\theta\left(b_{i}\right)=\overline{1} & i=1, \cdots, h \\
\theta\left(x_{\imath}\right)=\overline{1} & i=1, \cdots, p+1-2 q \\
\theta\left(e_{\imath}\right)=\theta\left(c_{\imath}\right)=\overline{1} & i=1, \cdots, q-2 h+1
\end{array}
$$

where $a_{\imath}, b_{i}, x_{\imath}, e_{\imath}, c_{i}$ are the canonical generators of $\Gamma_{1}$. Then, by [9], [5] and $[4], \operatorname{ker} \theta$ is an NEC groups with signature $(p+1 ;-;[-] ;\{-\})$. So, $D / \operatorname{ker} \theta$ is a non-orientable $q$-hyperelliptic KS without boundary with algebraic genus $p$ and its $q$-hyperellipticity group $\Gamma_{1}$ has signature (a).

Let us see 2). Given $1 \leqq h \leqq q$ (if $p$ is even) or $1 \leqq h \leqq q+1$ (if $p$ is odd) and an NEC group $\Gamma_{1}$ with signature (b), we define the epimorphism $\theta: \Gamma_{1} \rightarrow Z_{2}$ by means of

$$
\begin{array}{ll}
\theta\left(d_{\imath}\right)=\overline{0} & i=1, \cdots, h \\
\theta\left(x_{\imath}\right)=\overline{1} & i=1, \cdots,(p+1)-2 q \\
\theta\left(e_{\imath}\right)=\theta\left(c_{\imath}\right)=\overline{1} & i=1, \cdots, p-h+1
\end{array}
$$

where $d_{\imath}, x_{\imath}, e_{\imath}, c_{\imath}$ are the canonical generators of $\Gamma_{1}$. As in the previous case one can show that $D / \operatorname{ker} \theta$ is a surface we have looked for.

Proposition 2.3. Let $X=D / \Gamma$ be a non-orientable $q$-hyperelliptic $K S$ without boundary, with algebraic genus $p>4 q+1$. Then the automorphism of the hyperellipticity $\Phi$ is central and unique.

Proof. First we will show the uniqueness.

Let us suppose that there are two $q$-hyperellipticity groups $\Gamma_{1}$ and $\Gamma_{1}^{\prime}$. Then $\left[\Gamma_{1}: \Gamma\right]=2$ and $\left[\Gamma_{1}^{\prime}: \Gamma\right]=2$, and so $\left[\Gamma_{1}^{+}: \Gamma^{+}\right]=2$ and $\left[\Gamma_{1}^{\prime+}: \Gamma^{+}\right]=2$. Thus $\Gamma_{1}^{+} / \Gamma^{+}$and $\Gamma_{1}^{\prime+} / \Gamma^{+}$are groups of automorphisms of order two of $X^{+}=D / \Gamma^{+}$. Moreover both $D / \Gamma_{1}^{+}$and $D / \Gamma_{1}^{\prime+}$ have genus $q$. By hypothesis $p>4 q+1$, thus (see [1] and [2]) $\Gamma_{1}^{+}=\Gamma_{1}^{\prime+}$. Since $\Gamma$ has reversing-orientation elements, $\Gamma=\Gamma^{+} \cup g \Gamma^{+}$, where $g \in\left(\Gamma-\Gamma^{+}\right)$.

Since $\Gamma \subset \Gamma_{1}$ and $\Gamma \subset \Gamma_{1}^{\prime}$, we have $g \in \Gamma_{1}$ and $g \in \Gamma_{1}^{\prime}$. Thus $\Gamma_{1}=\Gamma_{1}^{+} \cup g \Gamma_{1}^{+}$ and $\Gamma_{1}^{\prime}=\Gamma_{1}^{\prime+} \cup g \Gamma_{1}^{\prime+}$. Hence $\Gamma_{1}=\Gamma_{1}^{\prime}$.

Now, we are going to see that $\Phi$ is central.

Let $\langle\Phi\rangle \cong \Gamma_{1} / \Gamma$, where $\Gamma_{1}$ is the group of the $q$-hyperellipticity of $D / \Gamma$, and let $\beta \in N_{G}(\Gamma) / \Gamma$. Then there exists $g \in N_{G}(\Gamma)$ such that, $\beta=g \Gamma$. From 
$\langle\Phi\rangle \cong \Gamma_{1} / \Gamma=\{\Gamma, l \Gamma\}$, we obtain

$$
\left\langle\beta \Phi \beta^{-1}\right\rangle=\left\{g \Gamma \Gamma \Gamma g^{-1}, g \Gamma l \Gamma \Gamma g^{-1}\right\}=\left\{\Gamma, g l g^{-1} \Gamma\right\}=\left(g \Gamma_{1} g^{-1}\right) / \Gamma .
$$

Thus since $\Gamma_{1}$ is isomorphic to $g \Gamma_{1} g^{-1}$ and $\Gamma$ is contained in both groups, the uniqueness of $\Gamma_{1}$ implies that $\Gamma_{1}=g \Gamma_{1} g^{-1}$ and so $\left\langle\beta \Phi \beta^{-1}\right\rangle=\langle\Phi\rangle$. Here by $\beta \Phi \beta^{-1}=\Phi$ and hence $\Phi$ is central in the full group of automorphism of $X$.

\section{Bounds for the order of the group of automorphisms.}

Let $X=D / \Gamma$ be a non-orientable $q$-hyperelliptic KS without boundary, and let $\Phi$ be an automorphism of the $q$-hyperellipticity. Then $\langle\Phi\rangle \cong \Gamma_{1} / \Gamma$ for a certain NEC groups $\Gamma_{1}$ and by (2.2) $X /\langle\Phi\rangle \cong D / \Gamma_{1}$ is an orientable KS with boundary or a non-orientable KS (with or without boundary).

In the next Theorem we are going to see that the bound $84(g-1)$ for the order of the group of the automorphisms of a non-orientable KS without boundary $X$ can be improved when $X$ is $q$-hyperelliptic and $X /\langle\Phi\rangle$ has non empty boundary.

THEOREM 3.1. Let $X=D / \Gamma$ be a non-orientable q-hyperelliptıc $K S$ without boundary of algebraic genus $p>4 q+1$.

Let $\Phi$ be the automorphism of the q-hyperellipticity. If $X /\langle\Phi\rangle$ is a surface with boundary then,

1) $\mid$ Aut $X \mid \leqq 12(p-1)$ (where Aut $X$ is the full group of automorphism of $X$ ).

2) If $\mid$ Aut $X \mid=12(p-1)$, then $X$ is a hyperelliptic surface of algebraic genus 2 and Aut $X \cong D_{6}$.

Proof. Since $X=D / \Gamma$ is a non-orientable KS without boundary of algebraic genus $p$, the signature of $\Gamma$ must be $(p+1 ;-;[-] ;\{-\})$. A group of automorphisms of $X$ can be represented as $\Gamma^{\prime \prime} / \Gamma$ where $\Gamma \triangleleft \Gamma^{\prime \prime}$.

$X$ is $q$-hyperelliptic and $p>4 q+1$. Thus by (2.2) and (2.3) there exists an unique NEC groups $\Gamma_{1}$ with signature

(a) $\left(h ;+;[2 \cdots \cdots \cdots \cdots \cdot 2] ;\left\{(-)^{q-2 h+1}\right\}\right), \quad 0 \leqq h \leqq q / 2$

or

(b) $\left(h ;-;[2 \cdots \cdots \cdots \cdots \cdot 2] ;\left\{(-)^{q-h+1}\right\}\right), \quad 1 \leqq h \leqq q$ if $p$ is even

$1 \leqq h \leqq q+1$ if $p$ is odd,

such that $\Gamma \triangleleft \Gamma_{1} \triangleleft \Gamma^{\prime \prime}$ and $\langle\Phi\rangle \cong \Gamma_{1} / \Gamma$. Since $X /\langle\Phi\rangle$ has boundary, $h \neq q+1$ in (b), and thus there are empty period cycles in the signature of $\Gamma_{1}$.

We are going to show that there is either an empty period cycles or a period cycle with two consecutive periods equals to ' 2 ' in the signature of $\Gamma$ ".

If this were not the case then from the normality of $\Gamma_{1}$ a reflection $c$ of canonical generators of $\Gamma^{\prime \prime}$ would belong to $\Gamma_{1}$. 
If this reflection $c$ belongs to a period cycle with unique period $n$, then the two reflections $c$ and $c^{\prime}$ that generate this period are conjugated. Thus by the normality the reflection $c^{\prime}$ also belongs to $\Gamma_{1}$. As a result the period $n$ must appear in a period cycle of $\Gamma_{1}$. This is a contradiction, because all period cycles of $\Gamma_{1}$ are empty. Thus we can assume that the reflection $c$ belongs to a period cycle having two consecutive periods $n^{\prime}$ and $n^{\prime \prime}$ one of which is different than 2. Without loss generality we can assume that this periods are associated to reflections $c, c^{\prime}, c^{\prime \prime}$, i. e.

$$
\left(c^{\prime} c\right)^{n^{\prime}}=\left(c c^{\prime \prime}\right)^{n^{\prime \prime}}=1 \text {. }
$$

If $c^{\prime}$ or $c^{\prime \prime}$ belong to $\Gamma_{1}$ then a period $n^{\prime}$ or $n^{\prime \prime}$ respectively appear in a period cycle of $\Gamma_{1}$. It is a contradiction as above.

If neither $c^{\prime}$ nor $c^{\prime \prime}$ belong to $\Gamma_{1}$ then by Theorem 1 of [5] the values $n^{\prime} / 2$ and $n^{\prime \prime} / 2$ must appear in period cycle of $\Gamma_{1}$. A contradiction appears once more.

Since $\mid$ Aut $X|=| \Gamma|/| \Gamma^{\prime \prime} \mid \geqq 12(p-1)$ and $|\Gamma|=2(p-1),\left|\Gamma^{\prime \prime}\right| \leqq \pi / 6$. From Section 1 (2) we have that the unique signature that has an empty period-cycle or a period-cycle with two consecutive periods equal to 2 and area smaller than $\pi / 6$ is

$$
(0 ;+;[-] ;\{(2,2,2,3)\}) .
$$

Thus $\mid$ Aut $X \mid \leqq 12(p-1)$ and if $\mid$ Aut $X \mid=12(p-1) \Gamma^{\prime \prime}$ has the signature (*).

Now, we are going to prove that if $\mid$ Aut $X \mid=12(p-1)$, then $p=2$ and Aut $X$ is $D_{6}$.

As $\Gamma_{1} \triangleleft \Gamma^{\prime \prime}$, there exists the epimorphism $\theta: \Gamma^{\prime \prime} \rightarrow \Gamma^{\prime \prime} / \Gamma_{1}$ with $\operatorname{ker} \theta=\Gamma_{1}$. If $c_{1}, c_{2}, c_{3}, c_{4}, c_{5}$ are the generators of $\Gamma^{\prime \prime}$ by [5] we have

$$
\begin{aligned}
& \theta\left(c_{1}\right)=x \\
& \theta\left(c_{2}\right)=y \\
& \theta\left(c_{3}\right)=1 \\
& \theta\left(c_{4}\right)=z \\
& \theta\left(c_{5}\right)=x,
\end{aligned}
$$

where $x, y, z$ are elements of order 2 in $\Gamma^{\prime \prime} / \Gamma_{1}$ and one of the following relations must be satisfied

i) $(x y)^{2}=(z x)^{3}=1$

ii) $x=y$ and $(z x)^{3}=1$.

If $i$ ) is satisfied, then by [4] there are no proper-periods in the signature of $\Gamma_{1}$, but $p>4 q+1$ and then the number of ' 2 ' in the proper-periods of (a) of (b) is $p+1-2 q>2 q+2>2$ and so i) can not be fulfiled.

Now assume that ii) is satisfied. Since $\left[\Gamma^{\prime \prime}: \Gamma_{1}\right]=6(p-1)$, by $[4]$ the number of ' 2 ' in the proper-periods of $\Gamma_{1}$ is $6(p-1) / 2$, and so $3(p-1)=p+1-2 q$. This equality is possible only in the case $p=2$ and $q=0$. Hence $h=0$ and $\Gamma_{1}$ 
would have a single cycle-period.

As $\Gamma^{\prime} / \Gamma_{1}$ has order 6 and it is a groups generated by $y, z$ with the relations $y^{2}=z^{2}=(y z)^{3}=1$, we have that $\Gamma^{\prime \prime} / \Gamma_{1} \cong D_{3}$; so that $\Gamma^{\prime \prime} / \Gamma$ would have order 12 and its quotient by a subgroups generated by a central element must be $D_{3}$. Thus $\Gamma^{\prime \prime} / \Gamma$ is $D_{6}$.

Finally, we need to prove that there exists a non-orientable hyperelliptic KS without boundary of algebraic genus 2 whose group of automorphism is $D_{6}$.

Let $\Gamma^{\prime \prime}$ be an NEC group with signature (*) and let $\theta$ be the epimorphism $\theta: \Gamma^{\prime \prime} \rightarrow D_{6}=\left\langle x, y \mid x^{2}=y^{2}=(x y)^{6}\right\rangle$ defined by

$$
\begin{aligned}
& \theta\left(c_{1}\right)=x \\
& \theta\left(c_{2}\right)=x(y x)^{3} \\
& \theta\left(c_{3}\right)=(x y)^{3} \\
& \theta\left(c_{4}\right)=x(y x)^{2} \\
& \theta\left(c_{5}\right)=x .
\end{aligned}
$$

Then by [9] and [4], $\operatorname{ker} \theta$ is an NEC group with signature $(3 ;-;[-] ;\{-\})$. Moreover, it is easy to check that $(x y)^{3}$ is a central element of $D_{6}$ and $c_{3}, c_{1} c_{2}$, $\left(c_{4} c_{5}\right)^{3}$ and $\left(c_{2} c_{4}\right)^{3}$ belong to $\theta^{-1}\left(\left\langle(x y)^{3}\right\rangle\right)$. By [9], [4] and [5] $\theta^{-1}\left(\left\langle(x y)^{3}\right\rangle\right)$ is an NEC group with signature $(0 ;+;[2,2,2] ;\{(-)\})$. Let us denote $\operatorname{ker} \theta$ by $\Gamma$ and $\theta^{-1}\left(\left\langle(x y)^{3}\right\rangle\right)$ by $\Gamma_{1}$. We have $\Gamma \triangleleft \Gamma_{1} \triangleleft \Gamma^{\prime \prime}$ and so $D / \Gamma$ is a non-orientable hyperelliptic KS without boundary of algebraic genus 2 having $D_{6}$ as the group of automorphisms.

If $p$ is even, then by Theorem $2.2 X /\langle\Phi\rangle$ is a surface with nonempty boundary and thus we have the following Corollary.

COROLlaRY 3.2. Let $X=D / \Gamma$ a non-orientable q-hyperelliptic $K S$ without boundary with algebraic genus $p>4 q+1$ and $p$ even, then:

1) $\mid$ Aut $X \mid \leqq 12(p-1)$

2) If $\mid$ Aut $X \mid=12(p-1)$, then $X$ is a hyperelliptic surface of algebraic genus 2 and Aut $X \approx D_{6}$.

Remark. By the functorial equivalence between the category of $\mathrm{KS}$ and the category of algebraic curves, established by Alling and greenleaf in [3], there is a correspondence between the non-orientable KS without boundary and the purely imaginary real curves (for example $x^{2}+y^{2}=-1$ corresponds to real projective plane). We say that a purely imaginary real curve $C$ is said to be $q$-hyperelliptic if and only if it admits an involution $\Phi$, such that if $\Phi^{\sim}$ is the extension of $\Phi$ to the complexification $C^{\sim}$ of $C$, then $C^{\sim} / \Phi^{\sim}$ has genus $q$. So all the results obtained above may be expressed in terms of purely imaginary real curves. 


\section{REFERENCES}

[1] AcColA, R.D.M., Riemann Surfaces with Automorphism Groups admitting partitions, Proceedings of American Math. Society, Vol. 21 (1969), 477-482.

[2] Accola, R. D. M., Strongly branched coverings of closed Riemann Surfaces, Proceedings of American Math. Society, Vol. 26 (1970), 315-322.

[3] Alling, N. L. And Greenleaf, N., Foundations of the Theory of Klein Surfaces, Lect. Notes in Math., 219 (1971), Springer.

[4] Bujalance, E., Proper periods of normal NEC Subgroups with even index, Rev. Mat. Hisp.-Amer. (4) 41 (1981), 121-127.

[5] Bujalance, J. A., Normal subgroups of even index in an NEC group, Arch. Math. 49 (1987), 470-478.

[6] Bujalance, E., Etayo, J.J. and Gamboa, J.M., Hyperelliptic Klein Surfaces, Quart. J. Math. Oxford, 36 (1985), 141-157.

[7] Bujalance, E., Etayo, J. J. and Gamboa, J. M., Superficies de Klein ElipticasHiperelipticas Memorias de R. Acad. Ciencias, tomo XII, 1985.

[8] Bujalance, E. And Etayo, J.J., A characterization of $Q$-Hyperelliptic compact planar Klein Surface, Abh. Math. Sem. Univ. Hamburg, to appear.

[9] Hoare, A.M.H. and Singerman, D., The orientability of Subgroups of plane Groups, London Math. Soc.: Lect. Notes Ser. 71 (1982), 221-227.

[10] Macbeath, A.M., The classification of non-Euclidean plane crystallographic groups, Canad. J. Math. 6, 1967.

[11] Maclachlan, C., Smooth covering of Hyperelliptic Surfaces, Quart. J. Math. Oxford, 22 (1971), 117-123.

[12] MAY, C. L., Large Automorphism Groups of Compact Klein Surfaces with boundary, Glasgow Math. J. 18, 1977.

[13] Singerman, D., On the structure of non Euclidean Crystallographic Groups, Proc. Cambridge Phil. Soc. 76 (1974), 233-240.

[14] Wilkie, M.C., On non Euclidean Crystallographic groups, Math. Zeit. 91 (1966), 87-102.

Depart. Matematicas Fundamentales

Facultad de Ciencias

U. N.E.D.

MADRID, SPAIN 